B. Schoser ${ }^{1}$, P. Baum ${ }^{2}$, M. Boentert ${ }^{3}$, K. Dillmann ${ }^{4}$, A. Emmer ${ }^{5}$, S. Knauss ${ }^{6}$, E. Enax-Krumova ${ }^{7}$, J. Grosskreutz ${ }^{8}$, A. Güttsches ${ }^{7}$, K. Hellwig ${ }^{9}$, K. Holzapfel ${ }^{10}$, C. Kornblum ${ }^{11}$, H. Lehmann ${ }^{12}$, A. Melms ${ }^{13,14}$, T. Meyer ${ }^{6}$, S. Petri ${ }^{15}$, L. Pilgram ${ }^{16}$, K. Reiners ${ }^{17}$, A. Saak ${ }^{18}$, J. Schäfer ${ }^{18}$, J. Schmidt ${ }^{19}$, C. Schneider-Gold ${ }^{9}$, M. Schons ${ }^{20}$, P. P. Urban ${ }^{21}$, M. Vorgerd ${ }^{7}$, P. Young ${ }^{22}$, S. Zierz ${ }^{5}$

${ }^{1}$ Friedrich-Baur-Institut, Neurologische Klinik und Poliklinik, Ludwig-Maximilians-Universität München, München, Deutschland

${ }^{2}$ Klinik und Poliklinik für Neurologie, Universitätsklinikum Leipzig, Leipzig, Deutschland

${ }^{3}$ Klinik für Neurologie mit Institut für Translationale Neurologie, Universitätsklinikum Münster, Münster, Deutschland

${ }^{4}$ Klinik für Neurologie, Universitätsklinikum Homburg/Saar, Homburg/Saar, Deutschland

${ }^{5}$ Klinik und Poliklinik für Neurologie, Universitätsklinikum Halle, Halle (Saale), Deutschland

${ }^{6}$ Neurologische Hochschulambulanz und ALS-Ambulanz, Charité - Universitätsmedizin Berlin, Berlin, Deutschland

${ }^{7}$ Neurologische Klinik und Poliklinik, BG-Universitätskliniken Bergmannsheil, Ruhr-Universität Bochum, Bochum, Deutschland

${ }^{8}$ Thüringer Neuromuskuläres Zentrum, Klinik für Neurologie, Universitätsklinikum Jena, Jena, Deutschland

${ }^{9}$ Klinik und Poliklinik für Neurologie, Katholisches Universitätsklinikum Bochum, Bochum, Deutschland

${ }^{10}$ Klinik für Neurologie und klinische Neurophysiologie, Universitätsklinikum Augsburg, Augsburg, Deutschland

${ }^{11}$ Klinik und Poliklinik für Neurologie, Sektion für Neuromuskuläre Erkrankungen, Universitätsklinikum Bonn, Bonn, Deutschland

${ }^{12}$ Klinik und Poliklinik für Neurologie, Universitätsklinikum Köln, Köln, Deutschland

${ }^{13}$ Neurologische Praxis Stuttgart, Stuttgart, Deutschland

${ }^{14}$ Zentrum für Neurologie, Universitätsklinikum Tübingen, Tübingen, Deutschland

${ }^{15}$ Klinik und Poliklinik für Neurologie, Medizinische Hochschule Hannover, Hannover, Deutschland

${ }^{16}$ Medizinische Klinik 2, Infektiologie, Universitätsklinikum Frankfurt, Frankfurt, Deutschland

${ }^{17}$ Neurologische Klinik, Hermann-Josef-Krankenhaus Erkelenz, Erkelenz, Deutschland

${ }^{18}$ Klinik und Poliklinik für Neurologie, Universitätsklinikum Dresden, Dresden, Deutschland

${ }^{19}$ Klinik für Neurologie, Neuromuskuläres Zentrum, Universitätsmedizin Göttingen, Göttingen, Deutschland

${ }^{20}$ Klinik I für Innere Medizin, Universitätsklinikum Köln, Köln, Deutschland

${ }^{21}$ Klinik und Poliklinik für Neurologie, Asklepios-Klinikum Hamburg-Barmbek, Hamburg, Deutschland

${ }^{22}$ Klinik für Neurologie Medical Park, Bad Feilnbach, Deutschland

\title{
Erratum zu: SARS-CoV-2/COVID-19 und neuromuskuläre Erkrankungen
}

\section{Bestandsaufnahme der DGN (Deutsche Gesellschaft für Neurologie) Kommission Motoneuron- und neuromuskuläre Erkrankungen}

\section{Erratum zu:}

DGNeurologie 2020

https://doi.org/10.1007/s42451-020-00198-2

Die ursprünglich publizierte Online-Version des Beitrags enthielt einen fehlerhaften Interessenkonflikt. Dies wurde im Originalbeitrag berichtigt. Bitte beachten Sie die korrigierte Version.

Wir bitten, den Fehler zu entschuldigen.
Korrespondenzadresse

Prof. Dr. B. Schoser

Friedrich-Baur-Institut, Neurologische Klinik und Poliklinik

Ludwig-Maximilians-Universität München Ziemssenstr. 1, 80336 München, Deutschland benedikt.schoser@med.uni-muenchen.de

Die Online-Version des Originalartikels ist unter https://doi.org/10. 1007/s42451-020-00198-2zu finden.

DGNeurologie 2020 3 (5): 472

https://doi.org/10.1007/s42451-020-00210-9

Online publiziert: 13. Juli 2020

(c) Springer Medizin Verlag GmbH, ein Teil von Springer Nature 2020 